\title{
A study of correlation between prosthetic breast reconstruction, antibiotic prophylaxis and surgical wound drains
} \author{
Raffaele Rauso ${ }^{3}$, Mario Cherubino ${ }^{4}$, Pier Camillo Parodi ${ }^{1}$ \\ ${ }^{1}$ Department of Plastic Reconstructive Surgery, University of Udine, 33100 Udine, Italy. \\ ${ }^{2}$ Department of Surgical and Morphological Sciences, University of Insubria, 21100 Varese, Italy. \\ ${ }^{3}$ Department of Dentistry, University of Foggia, 71121 Foggia, Italy. \\ ${ }^{4}$ Department of Biotechnology and Science of Life, University of Insubria, 21100 Varese, Italy.
}

Emanuele Rampino Cordaro1, Nicola Zerbinati², Alice Garzitto1, Daria Almesberger ${ }^{1}$, Michela Francescon¹,

Correspondence to: Dr. Emanuele Rampino Cordaro, Plastic Surgery Department, Santa Maria della Misericordia Hospital, Santa Maria della Misericordia Place 15, 33100 Udine, Italy. E-mail: emanuele.rampino@uniud.it

How to cite this article: Rampino Cordaro E, Zerbinati N, Garzitto A, Almesberger D, Francescon M, Rauso R, Cherubino M, Parodi PC. A study of correlation between prosthetic breast reconstruction, antibiotic prophylaxis and surgical wound drains. Plast Aesthet Res 2017;4:24-31.

Dr. Emanuele Rampino Cordaro was born in Udine (Italy) on 30th September, 1971 and graduated in Medicine and
Surgery at the University of Udine on September 1996. He was specialized in Plastic Reconstructive Surgery at the
University of Udine on November 2002. After several training experiences in Italy and abroad, he has been enrolled
at the University Hospital "Santa Maria della Misericordia" in Udine (Italy) on December 2005, as Consultant Plastic
Surgeon. He is author and co-author of several publications on national and international journals, covering the main
fields of plastic reconstructive and aesthetic surgery.

Article history:

Received: 10-07-2016

Accepted: 23-01-2017

Published: 28-02-2017

Key words:

Breast implant,

antibiotic prophylaxis,

drains,

infections,

breast reconstruction

\begin{abstract}
Aim: The primary aim of this investigation into the correlation between prosthetic breast reconstruction, antibiotic prophylaxis and surgical drains was to determine whether the short-term prophylaxis recommended in the current guidelines is in fact able to sterilize the peri-prosthetic pocket in patients undergoing prosthetic breast reconstruction via tissue expansion, permanent implant placement, or Becker implants, as well as augmentation to correct symmetry. Methods: A total of 96 women who had undergone prosthetic breast reconstruction surgery were considered. Patients were recruited from the Plastic Surgery Clinic, Gemona, and the Surgery Clinic, Udine, both affiliated with the Udine "Santa Maria della Misericordia" University Hospital between May 2013 and May 2014. All patients were administered the recommended short-term antibiotic prophylaxis, i.e. $2 \mathrm{~g}$ cephazolin (plus $1 \mathrm{~g}$ eventually given after $3.5 \mathrm{~h}$ of surgery) $30 \mathrm{~min}$ before surgery. Records pertaining to each patient were kept in a specific study chart. Results: Samples of peri-prosthetic drainage fluid were taken from $92.5 \%$ of the recipients of breast reconstruction/implant surgery. Only $2.3 \%$ of the samples analyzed were found to be positive for microbial strains. Conclusion: The results of this preliminary study are encouraging, demonstrating that the guidelines regarding short-term antibiotic prophylaxis are indeed effective.
\end{abstract}

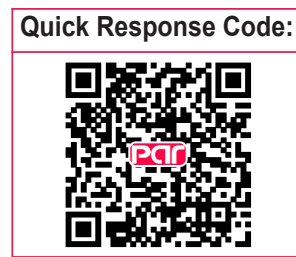




\section{INTRODUCTION}

The guidelines in use at the Udine S. Maria della Misericordia University hospital, which conform to the recommendations published by the Italian National Guidelines System (SNLG) and adopted by the FriuliVenezia-Giulia Regional Health Authority, recommend short-term pre-operative antibiotic prophylaxis via $2 \mathrm{~g}$ of cephazolin for breast implant patients, plus $1 \mathrm{~g}$ for operations scheduled to last more than $3.5 \mathrm{~h}$. The guidelines specify that antibiotic prophylaxis must be administered immediately before (in this case $30 \mathrm{~min}$ ) surgery, and be limited to the peri-operative period.

The literature indirectly confirms the efficacy of the short-term prophylaxis proposed in the guidelines, and published evidence supporting the superiority of prolonged prophylaxis is notably absent. ${ }^{[1-3]}$ Hence, based on the current evidence, extending prophylaxis to cover the first $24 \mathrm{~h}$ of the post-operative period can only be justified if there are major risk factors for postsurgical infection, and the reasons behind any decision to prolong prophylaxis beyond the recommended limit must be noted in the patient's medical records. ${ }^{[4,5]}$

Although we can assume that most, if not all, specialists in the sector adhere to such guidelines, the duration of antibiotic prophylaxis is nonetheless often the object of much discussion. Aside from the fear of peri-prosthetic infections, major concerns are also raised by the sequelae of infections, in particular implant loss, delays in neo-adjuvant therapy administration, unsatisfactory aesthetic outcomes and the need for further corrective surgery, not to mention potential medico-legal issues. ${ }^{[6,7]}$

Indeed, peri-prosthetic pocket infections tend to develop subclinically, leading to capsular contracture and other post-implant complications without overt signs or symptoms. However, peri-prosthetic discharge may represent an effective marker of even subclinical peri-prosthetic infection, and can be easily obtained from post-operative surgical drains.

Hence, in order to make a contribution, however minor, to this debate, the authors conducted a microbiological analysis of the peri-prosthetic discharge of breasts treated at Udine University Hospital. The aim was to establish objectively whether the short-term prophylaxis recommended in the guidelines is able to sterilize the peri-prosthetic pocket.

\section{METHODS}

Patients were recruited from the Plastic Surgery Clinic, Gemona, and the Surgery Clinic, Udine, both affiliated with the Udine "Santa Maria della Misericordia"
University Hospital between May 2013 and May 2014.

All study patients signed informed consent and gave their permission for publication of their pictures and samples analysis for research purpose; 86 patients $(92.5 \%)$ of the 96 considered.

Inclusion criteria consisted of all women undergoing breast implant surgery, comprising: (1) postmastectomy breast reconstruction via tissue expander/ implant or Becker expander; (2) expander/implant replacement surgery; and (3) breast augmentation to correct asymmetry.

The patient sample also included 3 cases of corrective surgery secondary to complications arising in the post-operative period, in particular: (1) 1 implant replacement with contralateral mastopexy following Becker expander rupture; (2) 1 implant replacement with latissimus dorsi myocutaneous flap following implant exposure; and (3) 1 latissimus dorsi myocutaneous flap reconstruction implant with breast implant and contralateral mastopexy following breast cancer relapse.

A total of 96 women who had undergone prosthetic breast reconstruction surgery were considered. In 50 patients, reconstruction was performed immediately after mastectomy (modified radical, nipple-sparing or skin-sparing) following a diagnosis of breast cancer. Surgery was performed to fit either a tissue expander or a permanent implant, accompanied or not by contralateral mastopexy. In an additional 12 patients, deferred post-mastectomy reconstruction via expander or permanent implant positioning, with or without latissimus dorsi flap reconstruction and/or contralateral mastopexy, was performed. In another group of 31 patients, expanders were replaced with permanent implants, with or without contralateral mastopexy and/or lipofilling. The remaining three patients underwent corrective surgery secondary to post-implant complications, specifically implant rupture, implant exposure, and breast cancer relapse, respectively.

All patients received the recommended short-term antibiotic prophylaxis, i.e. $2 \mathrm{~g}$ cephazolin (plus $1 \mathrm{~g}$ eventually given after $3.5 \mathrm{~h}$ of surgery) $30 \mathrm{~min}$ before surgery.

Records pertaining to each patient were kept in a specific study chart, the first part comprising the patient's personal information, diagnosis, lesion site, and type of surgery received. The second part of the study chart was used to compile data pertaining to the patient's "unnecessary" habits (smoking, drinking, 

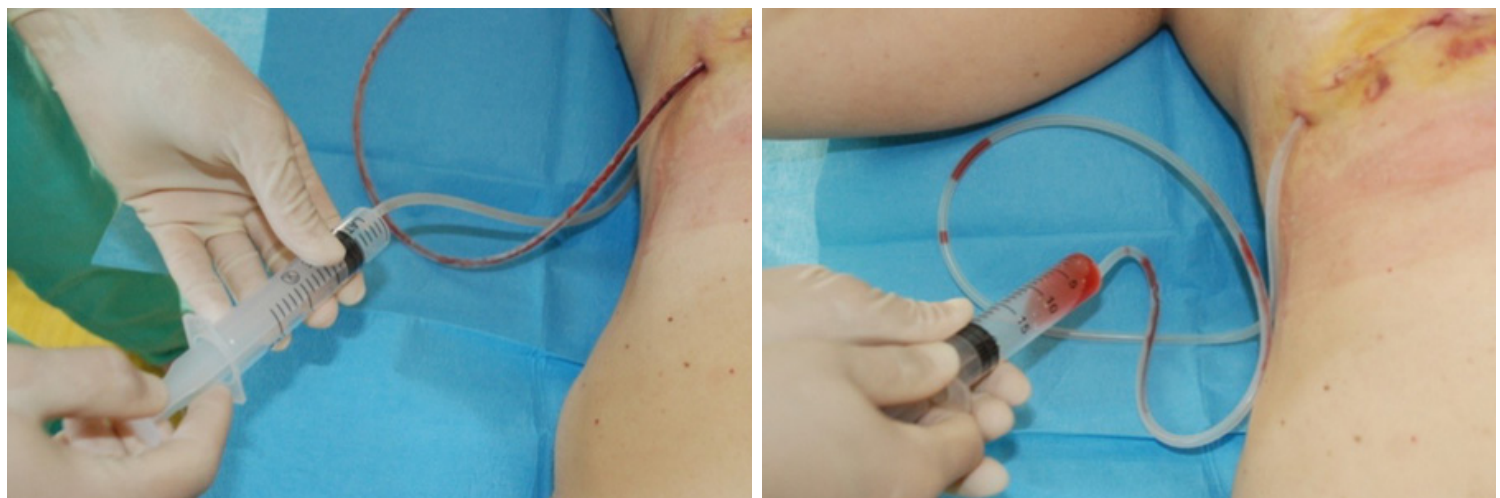

Figure 1: Aspirating fluid from the surgical drain
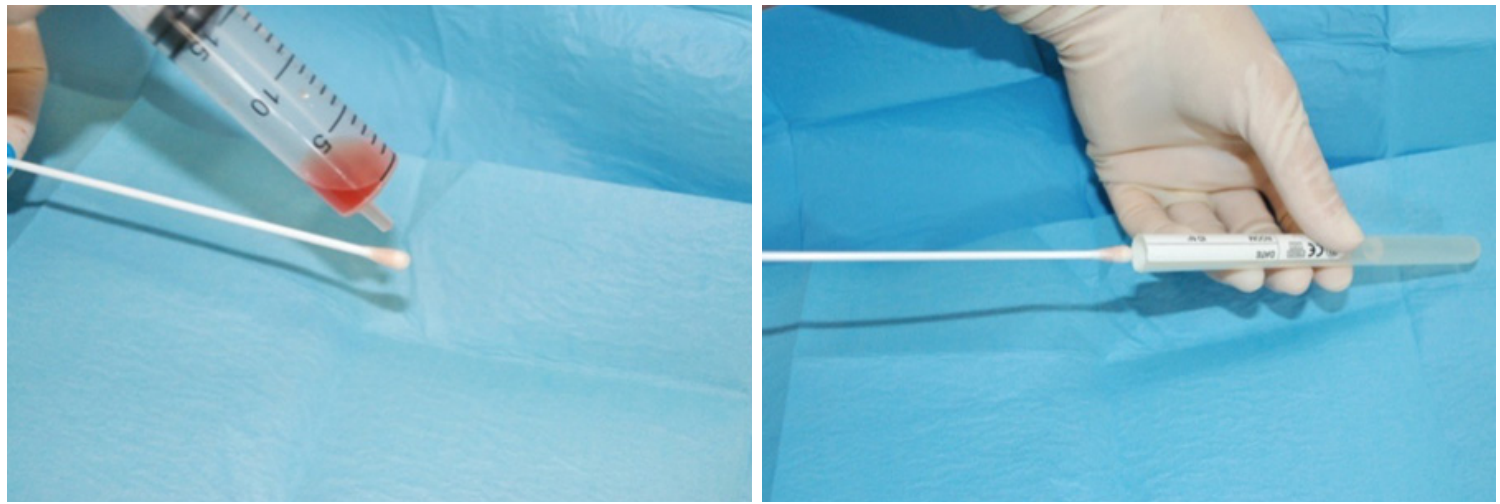

Figure 2: Preparing samples for microbiological testing

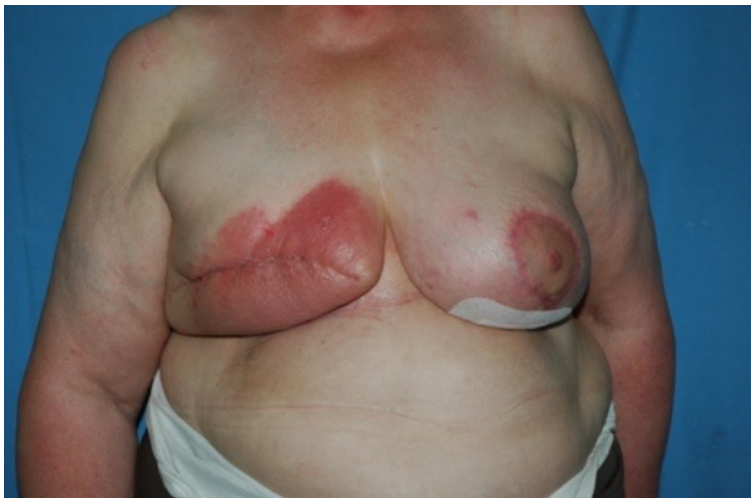

Figure 3: Evident signs of infection in the mastectomy/expander patient who tested positive for Staphylococcus aureus

not having a good diet, etc.), co-morbidities, and any medication or complementary treatment received or ongoing, while the third part was used to record the microbiological tests carried out, the results thereof, any microbial strain detected, and any signs of overt infection.

\section{RESULTS}

Samples of peri-prosthetic drainage fluid were taken from $86(92.5 \%)$ of the 96 recipients of breast reconstruction/implant surgery; the remaining $7.5 \%$ of patients did not adhere to the study. In this patient population the recommended short-term antibiotic prophylaxis was performed and the fluid collected was analyzed for microbiological contamination.

Samples were taken under sterile conditions by aspirating the peri-prosthetic fluid directly from the drain using a syringe, and transferring to sterile cotton swabs [Figures 1 and 2]. Samples were taken on the third day after surgery and upon drain removal (overall two times), and sent directly to the lab for testing.

Only 3 out of the 86 samples analyzed (2.3\%) were found to be positive for microbial strains, specifically Pseudomonas aeruginosa [Figures 3-5] and Propionibacterium acnes, respectively. In 1 case, a peri-prosthetic accumulation formed in the post-operative period was found to be positive for Staphylococcus aureus, but the drainage fluid taken from the same patient tested negative. In regards to the clinical and therapeutic characteristics of the patients who tested positive, the 55-year-old $S$. aureus patient, after mastectomy and expander placement, developed a fever, increased breast volume, and collection of pus, and the expander was therefore removed. The 45-year-old patient whose drainage fluid tested positive for Pseudomonas aeruginosa experienced 

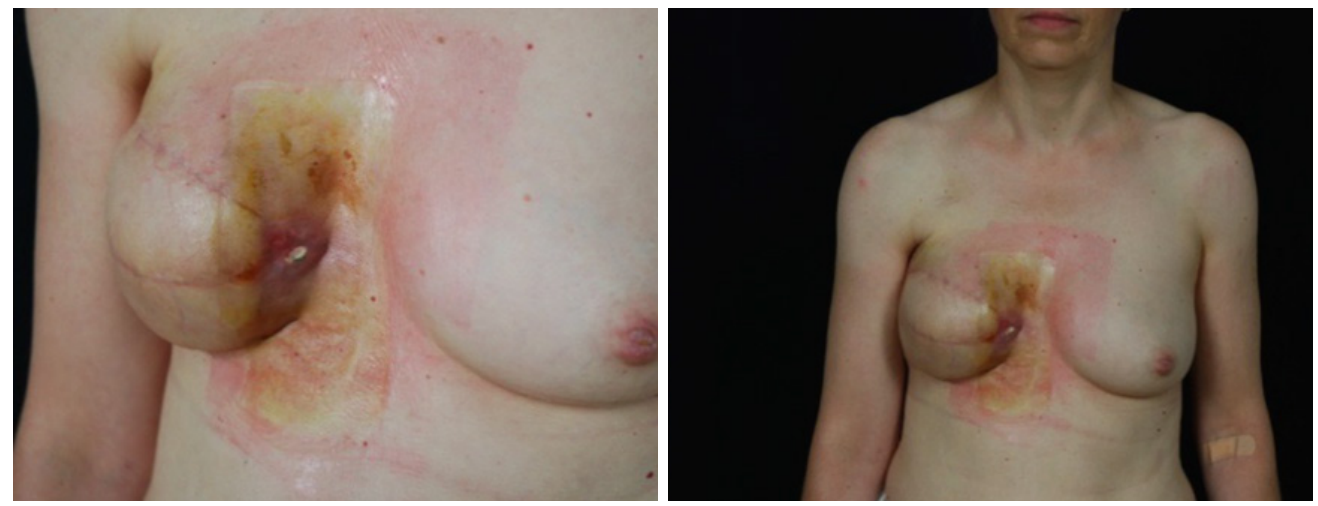

Figure 4: Latissimus dorsi myocutaneous flap failure and implant exposure in a patient who tested positive for Pseudomonas aeruginosa

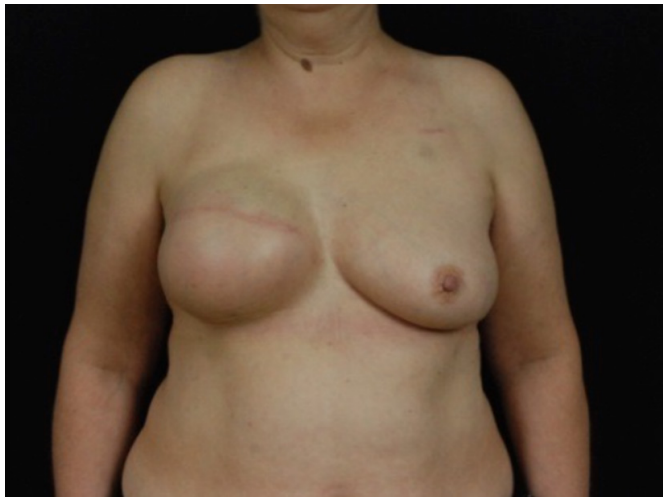

Figure 5: Patient positive for Propionibacterium acnes without clinical signs of infection

gradual loss of the apical portion of the skin paddle, originating at the medial apex, and consequent implant exposure following mastectomy and latissimus dorsi myocutaneous flap reconstruction. It is likely that the bacterial contamination of the peri-prosthetic environment was due to this exposure. Clinically, the patient demonstrated high inflammation indices and an accumulation of peri-prosthetic fluid, which was drained from the area of tissue loss.

In contrast, the 52-year-old mastectomy and implant reconstruction patient who tested positive for Propionibacterium acnes developed no clinical signs of infection, and completed the tissue expansion cycle with success. As no clinical signs of infection appeared during this cycle, the patient was not medicated, and the positive result was tentatively ascribed to contamination of the sample, pending further monitoring.

\section{DISCUSSION}

For each patient, including the three who tested positive for contamination, the variables associated in the literature with a greater risk of peri-prosthetic infection were analyzed. The first of these variables was age. The mean age of the 86 patients studied was
53 years. Forty-four out of 96 (45\%), i.e. the majority, fell into the range of 45 to 55 years of age. Ten $(20.8 \%)$ were in the age range 44 to 35 years, $18(18.7 \%)$ were between 65 and 74 years of age, and the remaining $14(14.5 \%)$ were aged between 55 and 64 years. There is no statistically significant correlation between advanced patient age and the risk of contracting a surgical site infection (SSI) in the literature, as the majority of studies show no statistically significant relationship between these 2 variables. ${ }^{[2,8]}$ In fact, rather than age, authors are more inclined to consider the presence of co-morbidities and the overall physical condition of the patient prior to surgery. Nevertheless, all three patients in the current study who had samples positive for microbial strains were of an intermediate age, with the patient infected by Staphylococcus aureus being 55 years old, the patient infected by Pseudomonas aeruginosa 45 years, and the patient infected by Propionibacterium acnes 52 years. The fact that these patients were not elderly confirms the widespread opinion in the literature that the risk of SSI onset is not conditioned by advanced age.

The second variable studied was smoking. Of the 86 patients, $14(16.2 \%)$ were smokers, smoking a mean of 10 cigarettes a day, and had been smoking for an average of 15 years. The literature contains many studies that demonstrate a statistically significant correlation between smoking and SSI risk, and the majority of authors contend that there is a statistically significant relationship between these 2 variables. ${ }^{[9,10]}$ However, these studies do suffer from a common limitation, namely that it is difficult to define terms like "regular" and "active" smoking statistically. In order to obtain statistically valid data on this variable, it would be necessary to adopt standardized measures of smoking history and find suitable controls to eliminate any bias.

Wound healing is dependent on the local blood supply, and smoking induces a state of chronic 
vasoconstriction, reducing blood vessel gauge and thereby reducing the flow of blood. Furthermore, the appearance of the scar is also adversely affected by smoking, and it is therefore strongly suggested that patients refrain from smoking in the 2-3 weeks before and after the operation. In the current study, however, none of the 3 women who tested positive admitted to smoking, and it is therefore impossible to confirm the literature findings in this sample.

The third variable considered in this study was the presence of co-morbidities. The majority of the 86 patients studied $(70 ; 81.4 \%)$ were not found to be affected by other pathologies. Only 10 patients $(11.6 \%)$ had metabolic issues or autoimmune disease; infectious diseases were not present. There was no comorbidity data available for 5 cases.

There are many studies in the literature that demonstrate a statistically significant correlation between the presence of co-morbidities in general and an increased risk of contracting an SSI. ${ }^{[11-13]}$ The National Nosocomial Infections Surveillance System considers three key variables to be independently associated with the risk of SSI, namely: (1) a wound infected or contaminated surgically; (2) the duration of the operation; and (3) an American Society of Anesthesiologists (ASA) Physical Status Classification score greater than 2 . Olsen et al. ${ }^{[14]}$ also demonstrated that an ASA score of 2 or 3 is associated with a statistically significant risk of developing an SSI.

Regarding metabolic function disorders, many researchers have set out to find a possible link between diabetes mellitus, for example, and the risk of peri-prosthetic infection. However, secondary to contrasting findings reported in the literature, this topic remains controversial. ${ }^{[8,14]}$ As none of the three patients who tested positive in our study suffered from any metabolic diseases, the authors can add little to this debate.

There is no data in the literature suggesting that autoimmune diseases may represent a possible risk factor for SSIs. Nevertheless, the potential role of the drugs used to treat these conditions, e.g. steroids, cytostatics and immunosuppressors (azathioprine, cyclosporine), have been widely studied. The findings of several of these studies ${ }^{[15-17]}$ appear to suggest that patients treated pre-operatively with immunosuppressant drugs or steroids do in fact run a greater risk of SSIs. However, none of the three patients who tested positive in the current study had any concomitant diseases, and therefore no conclusions can be drawn in this regard.
The fourth variable analyzed was the presence of any complementary treatments administered prior to surgery, and their various combinations (chemotherapy, radiotherapy, hormone therapy). The majority (72; $83.7 \%$ ) of the 86 patients in our sample had not received any complementary treatment, while the remaining 14 had a fairly uniform distribution in terms of the combination of possible treatment combinations.

Regarding radiotherapy (RT), the literature contains many studies which demonstratea significantcorrelation between preoperative RT and the development of an SSI. Indeed, RT can provoke skin damage by the occlusion or damage of the microvascular system and chromosomal damage to the fibroblasts, inhibiting stem cell replication, angiogenesis, and collagen production. This manifests during the final phases of treatment as hyperemia and inflammation of the skin. Although this is a frequent occurrence, it is easily remedied via suitable topical treatment, and seldom necessitates the interruption of treatment. Nonetheless, connective tissue may also be damaged, and in the later stages of RT, areas of sclerosis, fibrosis, and hypertrophy of the pectoral muscle may occur. By provoking tissue fibrosis and microvascular damage, therefore, RT may delay wound healing, and can be associated with dehiscence, necrosis and infection. Moreover, when RT precedes reconstructive surgery, post-actinic damage may complicate the tissue expansion necessary to house the implant, as well as increase the risk of periprosthetic fibrosis and capsular contracture. ${ }^{[8,14]}$

The role of preoperative chemotherapy (CT) as a potential peri-prosthetic infection risk factor has also been widely studied. Although contrasting results have been reported, CT is known to cause myelosuppression and neutropenia, and thereby potentially increase the risk of infection. ${ }^{[8,16]}$ In the current study, however, none of the 3 patients whose samples tested positive for microbial contamination had previously received CT. In fact, of these 3 cases, only 1 (infected by Pseudomonas aeruginosa) had undergone complementary treatment consisting of RT plus hormone therapy. Although the size of the sample does not enable meaningful statistical analysis, the influence of RT cannot be ruled out in this case.

The fifth variable considered was the use of postsurgical wound drains. Indeed, the drains themselves may represent a risk factor for infection by promoting biofilm formation on the surface of the device, which may in turn promote the transfer of bacteria into the wound. It is also possible to contaminate the lower end of the drain during disconnection and emptying, potentially causing intra-luminal infection and 
contamination of the wound. ${ }^{15,18,19]}$ Hence, although the drain performs an indispensable function, namely to eliminate accumulated serum from the wound site and thereby deprive endogenous pathogens of an excellent medium for proliferation, it can provide a route through which such pathogens can enter the body. ${ }^{[20-24]}$

As mentioned previously, the regional guidelines for antibiotic prophylaxis (AP) dictate the use of $2 \mathrm{~g}$ cephazolin (plus $1 \mathrm{~g}$ in operations lasting longer than $3.5 \mathrm{~h}$ ) solely before surgery. Cephazolin, the first choice antibiotic, is a cephalosporin that protects against a wide range of both gram-positive (staphylococcus strains, including aureus; coagulase-negative staphylococci, with the exception of methycillinresistant strains; beta-haemolitic streptococcus groups $\mathrm{A}$ and $\mathrm{B}$ ) and gram-negative ( $E$. coli and Klebsiella) bacteria. Cephalosporins are generally well-tolerated and inexpensive drugs with timedependent pharmacokinetics and a half-life of roughly $2 \mathrm{~h}$. They are considered high protein bonding (85\%), and provide excellent tissue distribution. Cephazolin, in particular, is one of the preferred options for clean surgery.

In cases of cephalosporin allergy, vancomycin or clindamycin are other viable options. Other drugs, such as ampicillin, amoxicillin, piperacillin, ampicillin/ sulbactam, and amoxicillin/clavulanic acid, are also widely used for surgical applications, and are very efficacious against enterococcus strains, albeit no more so than the cephalosporins in AP.

The study charts showed that all 86 were administered $2 \mathrm{~g}$ of cephazolin intravenously, $30 \mathrm{~min}$ before skin incision, with an additional $1 \mathrm{~g}$ in cases lasting longer than $3.5 \mathrm{~h}$. With this treatment, as already stated, 3 out of the 86 showed contaminated drainage secretion and 1 peri prosthetic infection but sterile drain.

The study charts confirmed adherence to the duration of AP suggested by both the literature and regional guidelines. Indeed, there is no statistical proof that longterm AP (for example until the time of drain removal) is any more efficacious at preventing SSIs. ${ }^{[4]}$ On the contrary, there are various reports that extending AP to cover tubes, drains and catheters is either useless ${ }^{[1,2,8]}$ or inadvisable. ${ }^{[25,26]}$

Despite this evidence, it has been reported that many hospitals, both in Italy and abroad, routinely use longterm AP in surgical cases. Perrotti et al., ${ }^{[6]}$ for example, state that over $50 \%$ of the plastic surgeons interviewed administer AP well beyond the operating time, and as many as $61 \%$ until drain removal. Although this behavior is not very judicious, it is understandable. Indeed, should a surgery patient fall prey to an SSI, there are many legal questions to consider, not to mention the clinical and financial consequences of their sequelae, which may include implant loss, delay in neo-adjuvant therapy, unsatisfactory or unsightly outcomes, and, as a consequence, prolonged hospital stays and even revision surgery. This represents a strong incentive for surgeons to administer postoperative AP, especially in patients with drains, in the hope that prolonging the course of AP will reduce the risk of SSI. The practice is even more common in immediate prosthetic reconstruction patients, with the fear of infectionrelated implant loss being the driving concern. ${ }^{[27,28]}$

However, the unrestrained use of antibiotics, perhaps fueled by the lack of prospective studies in the literature, has led to the development of methicillinresistant colonies of Staphylococcus epidermidis, and to an increase in the incidence of colitis secondary to Clostridium difficilis, in addition to side effects and secondary infections. Furthermore, antibiotics administered after wound closure seem to have no prophylactic effect on bacterial contamination during the surgery itself. ${ }^{[29,30]}$

Having administered short-term AP as per the recommended guidelines, $2(2.5 \%)$ out of the 80 patients drain secretion samples analyzed were found to be positive for microbial strains, specifically Pseudomonas aeruginosa in 1 case and Propionibacterium acnes in the other. The patient whose drain was found to be positive for Pseudomonas aeruginosa developed progressive loss of the apical portion of the skin paddle, originating at the medial apex, and consequent implant exposure in the postoperative period. This makes it likely that the route of the bacterial contamination was, in fact, the exposed implant. In contrast, the patient whose drainage secretion tested positive for Propionibacterium acnes developed no clinical signs of infection, and completed the weekly expansion cycle with no complications.

In a further patient, a positive result for Staphylococcus aureus was detected in a peri-prosthetic accumulation formed in the post-operative period, but the fluid taken from the drain of the same patient was found to be negative.

As contaminated drainage samples were found in only 2 out of the 86 patients, one conceivably attributable to implant exposure, this study appears to confirm the validity of the current guidelines regarding shortterm AP. Nevertheless, there are certain limitations 
to declare. First, as the operations were performed in more than one hospital, rigid standardization of the study protocol was not possible. Although the medical and nursing staff had been trained to adhere to the rules of the study, several (16) cases had to be excluded due to procedural inaccuracies. The major limitation of the study, however, was the small sample size, which prevents the conclusions from achieving statistical significance.

Nevertheless, patients have continued to be added to the study group, with a view to increasing the data available and addressing certain secondary objectives. Specifically, the authors wish to evaluate, via microbiological analysis of the peri-prosthetic pocket during revision surgery, whether subclinical colonization persists upon implant replacement, and whether or not bacterial colonization leads to a greater incidence of capsular contracture. It will also be interesting to follow the progress of the patient whose peri-prosthetic fluid tested positive for Propionibacterium acnes upon drain removal, despite an absence of signs of clinical infection. In particular, the patient will be monitored for any sign of capsular contracture, which would lend weight to the literature hypothesis that peri-prosthetic infection is an important risk factor for this event in the long term. Should contracture indeed occur, it will also be interesting to note whether the microbial species isolated from the capsule is the same as that present in the drain fluid.

Despite the above-mentioned limitations, the results of this preliminary study are encouraging, demonstrating that the guidelines regarding short-term AP are indeed effective. However, it remains to be demonstrated that not prolonging prophylaxis does not statistically increase the risk of surgical failure, and does not therefore expose either the patient or surgeon to the burden of complications, whether major or minor.

\section{Financial support and sponsorship}

None.

\section{Conflicts of interest}

There are no conflicts of interest.

\section{Patient consent}

All patients signed informed consent.

\section{Ethics approval}

The Institutional Review Board approved this study.

\section{REFERENCES}

1. Throckmorton AD, Boughey JC, Boostrom SY, Holifield AC, Stobbs
MM, Hoskin T, Baddour LM, Degnim AC. Postoperative prophylactic antibiotics and surgical site infection rates in breast surgery patients. Ann Surg Oncol 2009;16:2464-9.

2. Khan UD. Breast augmentation, antibiotic prophylaxis, and infection: comparative analysis of 1,628 primary augmentation mammoplasties assessing the role and efficacy of antibiotics prophylaxis duration. Aesthetic Plast Surg 2010;34:42-7.

3. Hedick TL, Smith PW, Gazoni LM, Sawyer RG. The appropriate use of antibiotics in surgery: a review of surgical infections. Curr Probl Surg 2007;44:635-75.

4. Page CP, Bohnen JM, Fletcher JR, McManus AT, Solomkin JS, Wittmann DH. Anti-microbial prophylaxis for surgical wounds. Guidelines for clinical care. Arch Surg 1993;128:79-88.

5. Rohrich RJ, Rios JL. The role of prophylactic antibiotics in plastic surgery: whom are we treating? Plast Reconstr Surg 2003;112:617-8.

6. Perrotti JA, Castor SA, Perez PC, Zins JE. Antibiotic use in aesthetic surgery: a national survey and literature review. Plast Reconstr Surg 2002;109:1685-93; discussion 1694-5.

7. Lyle WG, Outlaw K, Krizek TJ, Koss N, Payne WG, Robson MC. Prophylactic antibiotics in plastic surgery: trends of use over 25 years of an evolving specialty. Aesth Surg J 2003;23:177-83.

8. Nahabedian MY, Tsangaris T, Manson PN. Infectious complications following breast reconstruction with expanders and implants. Plast Reconstr Surg 2003;112:467-76.

9. Cordeiro PG, McCarthy CM. A single surgeon's 12-year experience with tissue expander/implant breast reconstruction: part I. A prospective analysis of early complications. Plast Reconstr Surg 2006;118:825-31.

10. Ernori TG, Gaynes RP. An overview of nosocomial infections, including the role of the microbiology laboratory. Clin Microbiol Rev 1993;6:428-42.

11. Geubbels EL, Grobbee DE, Vandenbroucke-Grauls CM, Wille JC, de Boer AS. Improved risk adjustment for comparison of surgical site infection rates. Infect Control Hosp Epidemiol 2006;27:1330-9.

12. Clegg HW, Bertagnoll P, Hightower AW, Baine WB. Mammaplastyassociated mycobacterial infection: a survey of plastic surgeons. Plast Reconstr Surg 1983;72:165-9.

13. Courtiss EH, Goldwyn RM, Anastasi GW. The fate of breast implants with infection around them. Plast Reconstr Surg 1979;63:812-6.

14. Olsen MA, Lefta M, Dietz JR, Brandt KE, Aft R, Matthews R, Mayfield J, Fraser VJ. Risk factors for surgical site infection after major breast operation. J Am Coll Surg 2008;207:326-35.

15. Donlan RM, Costerton JW. Biofilms: survival mechanisms of clinically relevant microorganisms. Clin Microbiol Rev 2002;15:16793.

16. Yule GJ, Concannon MJ, Croll G, Puckett CL. Is there liability with chemotherapy following immediate breast reconstruction? Plast Reconstr Surg 1996;97:969-73.

17. Haley RW, Culver DH, Morgan WM, White JW, Emori TG, Hooton TM. Identifying patients at high risk of surgical wound infection. A simple multivariate index of patient usceptibility and wound contamination. Am J Epidemiol 1985;121:206-15.

18. Felippe WA, Werneck GL, Santoro-Lopes G. Surgical site infection among women discharged with a drain in situ after breast cancer surgery. World J Surg 2007;31:2293-9; discussion 2300-1.

19. Raves JJ, Slifkin M, Diamond DL. A bacteriologic study comparing closed suction and simple conduit drainage. Am J Surg 1984;148:61820.

20. McCarthy CM, Mehrara BJ, Riedel E, Davidge K, Hinson A, Disa JJ, Cordeiro PG, Pusic AL. Predicting complications following expander/ implant breast reconstruction: an outcomes analysis based on pre operative clinical risk. Plast Reconstr Surg 2008;121:1886-92.

21. McCarthy CM, Disa JJ, Pusic AL, Mehrara BJ, Cordeiro PG. The 
effect of closed suction drains on the incidence of local wound complications following tissue expander/implant reconstruction: a cohort study. Plast Reconstr Surg 2007;119:2018-22.

22. Puttawibul P, Sangthong B, Maipang T, Sampao S, Uttamakul P, Apakupakul N. Mastectomy without drain at pectoral area: a randomized controlled trial. J Med Assoc Thai 2003;86:325-31.

23. Soon PS, Clark J, Magarey CJ. Seroma formation after axillary lymphadenoctomy with and without the use of drains. Breast 2005;14:103-7.

24. Saratzis A, Soumian S, Willetts R, Rastall S, Stonelake PS. Use of multiple drains after mastectomy is associated with more patient discomfort and longer post operative stay. Clin Breast Cancer 2009;9:243-6.

25. Vilar-Compte D, Jacquemin B, Robles-Vidal C, Volkow P. Surgical site infections in breast surgery: case-control study. World J Surg 2004;28:242-6.

26. Mangram AJ, Horan TC, Pearson ML, Silver LC, Jarvis WR.
Guideline for Prevention of Surgical Site Infection, 1999. Centers for Disease Control and Prevention (CDC) Hospital Infection Control Practices Advisory Committee. Am J Infect Control 1999;27:97-132; quiz 133-4; discussion 96.

27. Clayton JL, Bazakas A, Lee CN, Hultman CS, Halvorson EG. Once is not enough: withholding postoperative prophylactic antibiotics in prosthetic breast reconstruction is associated with an increased risk of infection. Plast Reconstr Surg 2012;130:495-502.

28. Pajkos A, Deva AK, Vickery K, Cope C, Chang L, Cossart YE Detection of subclinical infection in significant breast implant capsules. Plast Reconstructr Surg 2003;111:1605-11.

29. Deva AK, Chang LC. Bacterial biofilms: a cause for accelerated capsular contracture? Aesthetic Surg J 1999;19:130-3.

30. Allweis TM, Boisvert ME, Otero SE, Perry DJ, Dubin NH, Priebat DA. Immediate reconstruction after mastectomy for breast cancer does not prolong the time to starting adjuvant chemotherapy. Am J Surg 2002;183:218-21. 